\title{
Plataformas Coworking
}

\author{
Coworking Platforms \\ Dr. Jesús SÁNCHEZ COTOBAL \\ Universidad Francisco de Vitoria \\ j.sanchez.prof@ufv.es
}

Resumen: El coworking es un nuevo ecosistema de trabajo que está revolucionando la forma en la que los diferentes profesionales llevan a cabo su trabajo, ya que se basa en compartir un nuevo espacio sin que cada uno pierda su independencia. La esencia del coworking se basa en hablar, compartir, conectar, proponer, unir y ayudar. Y por eso el coworking ha funcionado tan bien, porque todos los seres humanos necesitamos desarrollarnos en comunidad, compartiendo, interactuando y creando cosas en conjunto que no seríamos capaces si lo intentáramos de forma individual.

Abstract: Coworking is a new work ecosystem that is revolutionizing the way in which different professionals carry out their work, since it is based on sharing a new space without each losing their independence. The essence of coworking is based on speaking, sharing, connecting, proposing, uniting and helping. And that's why coworking has worked so well, because all human beings need to develop in community, sharing, interacting and creating things together that we would not be able to if we tried it individually.

Palabras clave: coworking, community builder, coworkers, networking, hubs, hackerspaces, freelances, desing thinking

Keywords: coworking, community builder, coworkers, networking, hubs, hackerspaces, freelances, desing thinking

\section{Sumario:}

\section{Introducción.}

II. Historia del coworking. 
III. Coworking.

3.1. Definición.

3.2. Modelo dominante Vs. Economía colaborativa.

3.3. Espacios para que funcione el coworking.

3.4. Ventajas del coworking.

3.5. Inconvenientes del coworking.

3.6. Conclusión parcial.

3.7. Generación de conocimientos y referentes.

3.8. Filosofía del coworking.

IV. Personas usuarias Vs. público objetivo

V. Mejores espacios del coworking en el mundo.

VI. Razones para trabajar en un espacio coworking.

VII. Marketing y el coworking.

VIII. Financiación y rentabilidad.

IX. El coworking en sucursales bancarias.

X. Conclusión.

XI. Webgrafía.

Recibido: septiembre de 2019.

Aceptado: noviembre de 2019. 


\section{INTRODUCCIÓN}

Este trabajo va a consistir en explicar el fenómeno coworking ya que se trata de un concepto bastante nuevo debido a la escasez de puestos de trabajo y por tanto se deben buscar alternativas viables para su realización. El espacio en el que se desarrolla esta forma de trabajo puede ser físico o virtual, pero ha de haber una comunidad existente a la que pertenecer, sin que sea condición necesaria participar activamente. También debe existir la figura de un community builder, el cual se encarga de velar por la comunidad ${ }^{1}$. Un community builder es un manager encargado de gestionar los espacios dedicados a esta actividad. Su función no consiste sólo en contribuir a su creación, sino que también debe preocuparse por su cuidado y su mantenimiento para aportar valor añadido. Este es el punto por el que separamos otras prácticas como hubs, oficina compartida o centro de negocios. A menudo los centros de negocio pueden tener un modelo mixto con coworking, donde los freelances alquilan el espacio para usar sus instalaciones, pero sin que se generen sinergias o sin implicarse en el centro. En línea con términos que se diferencian del coworking, también existen los hubs de emprendimiento, que son centros donde los emprendedores trabajan juntos, colaboran y forman una comunidad. A veces pueden llegar incluso a convertir las ciudades donde se instalan en punteros de emprendimiento, podemos decir que son como laboratorios de innovación. Un ejemplo en la práctica es el que está llevando a cabo BBVA con su programa de emprendimiento social con los impact hub de diferentes ciudades, en donde se han dado formaciones y sesiones didácticas fomentando el networking. Se trata de un programa de emprendimiento para jóvenes entre 18 y 29 años con un premio otorgado entre los programas que sean presentados. Cuando hablamos de lo primero que nos viene en mente es el conocido Silicon Valley, en EEUU, pero también hay muchos otros por todo el mundo. Volviendo al concepto de community builder, esta figura debe contar con partners, alianzas o sponsors que aporten valor al espacio mediante pagos en especie. También debe ser una persona proactiva, con buen manejo de las relaciones sociales, motivadora y conciliadora para construir relaciones en toda la comunidad y conocer cada uno de los perfiles que la integran y abierta a escuchar todas las propuestas de los mismos ${ }^{2}$.

\footnotetext{
${ }^{1} \mathrm{https}: / /$ www.muypymes.com/2016/02/11/utopic_us.

${ }^{2} \mathrm{https}: / /$ definicion.de/freelance/
} 
La esencia del coworking se basa en hablar, compartir, conectar, proponer, unir y ayudar. Y por eso el coworking ha funcionado tan bien, porque todos los seres humanos necesitamos desarrollarnos en comunidad, compartiendo, interactuando y creando cosas en conjunto que no seríamos capaces si lo llevásemos a cabo de forma individual. Todos los espacios en los que se desarrolla el coworking tienen que cumplir con una serie de normas para su buen funcionamiento, como zonas comunes donde se puedan relacionar todos los coworkers, libertad de acceso una vez se consiga el acceso a esta comunidad, el gestor ha de comportarse como un nexo entre los miembros, diferentes precios en función de la modalidad escogida, evolución, dinamismo y rapidez para llevar a cabo mejoras entre otras.

Hay empresas que "bautizan" como coworkings a nuevos espacios donde poder encontrar un alquiler más barato e interactuar como profesionales. Esto sería una salida a la precariedad que hubo, sobre todo, tras la crisis que sufrimos en 2008, lo cual no es una mala alternativa, pero pone en peligro el concepto puro de coworking, ya que el coworking surge de una realidad cambiante, y de ahí la necesidad de interactuar con otros profesionales intercambiando opiniones, creando nuevas ideas y formando una nueva comunidad. Hay un dato curioso que nos gustaría destacar, y es que "las mejores historias del coworking se han gestado con un café en la mano", es una de las mejores prácticas útiles para hacer nuevos contactos y compartir experiencias, lo que se traduce en tiempo intercambiado y compartido con los demás. Dedicar tiempo a otras personas, y no tanto a focalizar al máximo nuestro trabajo individual, es beneficioso para desarrollarnos como verdaderos profesionales.

El coworking se basa en el pago de una cuota diaria, mensual, por horas para poder disfrutar de todos los beneficios que ofrece aunque sea por tiempo limitado, pero durante todo ese tiempo se disfruta al 100\% de los servicios ofrecidos. Por tanto, el coworking es una forma de trabajo, pero trabajo grupal ya sea de la misma categoría o de otra diferente, obteniendo resultados satisfactorios por la combinación de maneras de pensar, trabajar, decidir, proponer, etc. Es una técnica muy útil porque cada con su asertividad propone sus aptitudes y conocimientos para enriquecer el proceso. En definitiva la filosofía del coworking se traduce en "juntos pensamos mejor". El coworking no es solo un espacio de trabajo, sino que también busca la creación de sinergias con otras personas, para que así se mejore el entorno laboral y social buscando oportunidades a través de las fortalezas del networking, ya que no sólo busca que las personas estén continuamente trabajando, sino que se relacionen entre ellas y puedan auto-formarse para lograr mejores objetivos. Ya que todos ellos tienen un mismo propósito que es desarrollar su actividad. Trabajar así es muy útil porque rompes con la rutina diaria. Como dice Utopicus: "Significa trabajar entre amigos, 
algo que surge de manera natural sin elegirlo, dando lugar al aumento de la capacidad de trabajo y la efectividad del mismo gracias a la atmósfera de sinergia y colaboración que los coworkers crean entre ellos"3

Existen diferentes criterios a la hora de elegir el tipo de coworking que mejor se adapte a nuestras necesidades:

a. Por precio. Bajo mi punto de vista, es normal que éste sea un factor importante que considerar, ya que a todos nos gusta pagar un precio por alquiler lo más bajo posible, pero quizá no encontraremos un retorno a nuestra inversión si no tenemos en cuenta otros criterios que pueden aportar más a nuestro enriquecimiento, como los que se enuncian a continuación.

b. Por proximidad. La cercanía a los lugares donde realizamos nuestra vida cotidiana es fundamental para compatibilizar el trabajo con una vida flexible.

c. Por ambiente de trabajo. Es fundamental visitar en persona el espacio antes de adentrarnos en la comunidad, para que este sea el último factor decisivo a la hora de elegir.

d. Por servicio. Cuando visitemos un espacio de coworking deberemos valorar detalles en el servicio que se ofrece: personal, decoración, rotación de personas, amabilidad y comodidad, etc.

e. Por la comunidad. A mi juicio es el factor más importante a considerar si queremos que sea una experiencia de provecho.

\section{HISTORIA DEL COWORKING}

$\mathrm{Su}$ origen se remonta a los lugares físicos donde los primeros hacerkerspaces ${ }^{4}$ trabajaban en la C-Base de Berlín, sobre los últimos años del siglo XX, concretamente en 1995. Más adelante Bernie Dekoven introdujo por primera vez el término coworking para denominar al espacio donde los trabajadores se juntaban para hacer trabajo colaborativo con ordenadores. A continuación, esta forma de trabajo fue expandiéndose por toda la cultura occidental. El famoso 42 West 24, en Nueva York, es una oficina que todavía hoy continúa

\footnotetext{
${ }^{3}$ https://www.utopicus.es.

${ }^{4}$ https://hipertextual.com/2011/05/hackerspaces-refugios-de-creatividad-y-conocimiento.
} 
en funcionamiento. También Viena fue partícipe del primer espacio europeo de trabajo compartido, llamado "Community Center for Enterpreneurs". Fue en San Francisco donde se abrió el primer centro de coworking oficial como iniciativa sin ánimo de lucro con wifi gratuito, almuerzos y comidas compartidas., dos días a la semana y descansos con meditación. La mayor red de espacios de coworking es llamada "Hub". Fue en Londres, en Angel Station y en Alemania, con las primeras cafeterías que hacían que la navegación a través de la red para sus clientes fuese de acceso gratuito, transformándose así en espacios de trabajo cooperativo. La creación de Google favoreció el coworking porque era navegación por la red sin necesidad de abonar nada.

Hoy en día, encontramos aproximadamente 3.000 espacios de trabajo colaborativo asignados por 80 países todos ellos desarrollando este fenómeno.

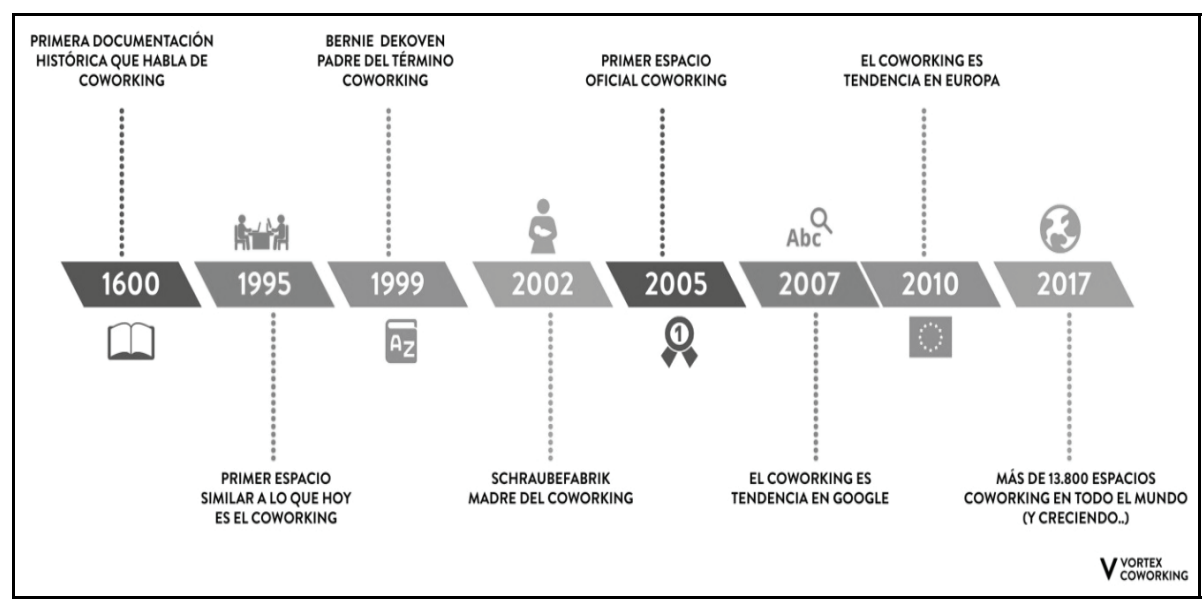

\section{COWORKING}

\subsection{Definición}

Coworking en español significa trabajo cooperativo, es un concepto innovador de trabajo en un mismo lugar permitiendo trabajar de forma independiente, realizando trabajos de manera autónoma, es lo que se llama, freelances ${ }^{5}$. Lo que busca el coworking es que autónomos, expertos, técnicos, pymes, etc. compartan conocimientos y ambientes, además de forma física y virtual, esto fomenta los proyectos empresariales que se les propongan, a pesar de no trabajar en lo mismo.

\footnotetext{
${ }^{5} \mathrm{http}: / /$ www.comunidadcoworking.es/que-es-coworking/.
} 
Este concepto no tiene un patrón definido, ya que en ocasiones se basa en dar tranquilidad a sus integrantes, mientras que otras veces se basa en movimiento y en que todo el mundo sepa manejar y aportar know how en cualquier proyecto en el que se involucre. Observamos como puede ser útil no solo para la empresa, sino que también para nuestro desarrollo personal y profesional, porque puede ampliar tu cartera de clientes y puedes cada día estar en un proyecto y con diferentes personas, obtener contactos, además de conocer un nuevo ambiente de trabajo.

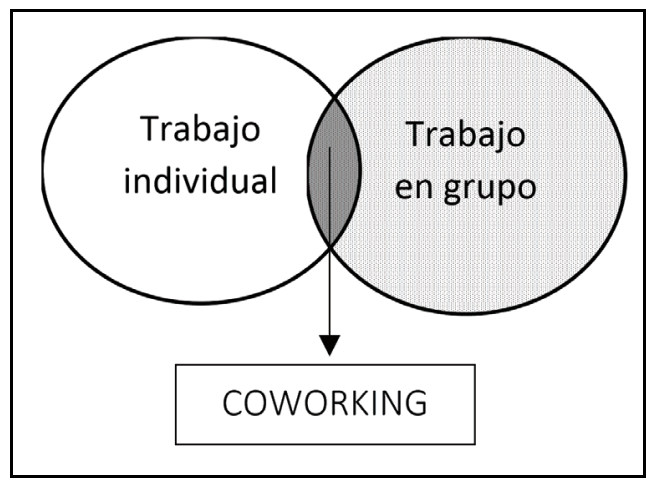

Los coworkers, describen el coworking como un estilo de trabajo en el que expertos autónomos comparten una atmósfera de trabajo, aunque actúan de manera independiente en el mundo de los negocios ${ }^{6}$. Ocurre cuando personas con talento y creativas comparten el mismo espacio físico, siendo éste una especie de local low-cost con conocimiento, donde comparten oportunidades de negocio, una manera de facilitar la colaboración formal e informal, suponiendo una evolución lógica que freelancer, emprendedores y autónomos venían demandando. Cuando hablamos de coworking, se nos viene a la cabeza la Economía Colaborativa, la cual está compuesta por tres elementos:

1. El avance tecnológico.

2. Las presiones económicas actuales que producen enfrentamientos entre los negocios $\mathrm{y}$,

3. El imperativo humano por la simplicidad. Con esto podemos hablar del fenómeno en el que un conjunto de personas comparte espacios para reducir costes compartiendo comodidad y equipamientos, para tener acceso a una agrupación de emprendedores para buscar colaboración dentro y fuera de diversos campos.

\footnotetext{
${ }^{6} \mathrm{https} / / /$ www.skyscanner.es/noticias/inspiracion/los-15-espacios-de-coworking-mas-cooldel-mundo.
} 
3.2. Modelo dominante VS. Economía colaborativa (Coworking)

\begin{tabular}{|l|l|l|l|}
\hline \multicolumn{4}{|c|}{ MODELO DOMINANTE } \\
\hline Producción & \multicolumn{1}{|c|}{ Distribución } & Intercambios & \multicolumn{1}{c|}{ Consumo } \\
\hline -Planificación & -Arriba/abajo & -Contratado & $\begin{array}{l}\text {-Estimulado por } \\
\text { productores }\end{array}$ \\
-Estandarización & -Poco para muchos & $\begin{array}{l}\text {-Sistema } \\
\text { Financiero } \\
\text { complejo } \\
\text {-Protección con } \\
\text { patentes }\end{array}$ & $\begin{array}{l}\text {-Cadena de } \\
\text { el acceso }\end{array}$ \\
\hline
\end{tabular}

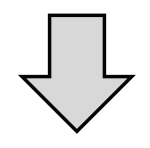

\begin{tabular}{|l|l|l|l|}
\hline \multicolumn{4}{|c|}{ ECONOMÍA COLABORATIVA } \\
\hline Producción & \multicolumn{1}{|c|}{ Distribución } & \multicolumn{1}{c|}{ Intercambios } & \multicolumn{1}{c|}{ Consumo } \\
\hline -Colaborativa & -P2P & -Basado en la confianza & $\begin{array}{l}\text {-Simulado por } \\
\text { la necesidad }\end{array}$ \\
-Por uno mismo & -Muchos para muchos & -Facilitado por TICS & $\begin{array}{l}\text {-Acceso sobre } \\
\text { la posesión }\end{array}$ \\
& $\begin{array}{l}\text {-Cadena de } \\
\text { suministro corto }\end{array}$ & $\begin{array}{l}\text {-Importancia del } \\
\text { valor de cambio }\end{array}$ \\
\hline
\end{tabular}

\subsection{Espacios para que funcione el coworking}

Los resumo básicamente en:

- Evolución y rapidez continua en el momento de ejecutar mejoras

- Convivencia y respeto.

- Zonas comunes que permiten la conexión entre coworkers.

- Autonomía de acceso.

- La figura del gestor del espacio como vínculo de unión entre coworkers.

- Particularidad sencilla de precios enfocadas al perfil-target elegido.

- Ideas que aporten eficacia y ayuden a promover proyectos. 


\subsection{Ventajas del coworking}

El provecho de poder formar parte de un espacio de trabajo cooperativo es sin duda de gran alcance para todas las personas que necesiten dar forma a sus ideas de negocio, debido a que las personas que acuden a estos lugares disponen de la misma intención que el resto de los participantes ${ }^{7}$. Cada una de estas personas busca lograr su sueño, por lo que se han encontrado diversidad de obstáculos por el camino y experiencias que les han hecho proseguir, todo esto hace que a las personas nuevas que acudan puedan explicarles sus vivencias y ocurrencias para que juntas puedan lograr los objetivos que se proponen. Algunos de los beneficios que se adquieren al pertenecer a estos espacios son:

- Perfecciona la disciplina y fija horarios.

- Implementa nuevos proyectos con otros coworkers. Cuando una persona es adulta se piensa que todos los círculos de amigos ya están cerrados y son imposibles de expandir. Por eso estas personas utilizan estas oficinas además de como herramienta profesional como elemento social con el que pueden ganar relaciones más que profesionales. El trabajo suele ser más ameno con amigos que sin ellos. Además, está demostrado que la gente es más productiva y colaborar de mejor manera cuando trabaja rodeada de gente y sobre todo si les cae bien. La conversación fomenta la creatividad y más aún cuando las ideas sean compartidas.

- Ayuda a sentirse protegido por otros profesionales y dejar a un lado la autonomía.

- Emprender a un coste reducido.

- Beneficios flexibles.

- Conectividad.

- Asistencia técnica.

- Ubicación.

- Red de apoyo. Lo profesionales autónomos consideran estos espacios como "salas de motivación." Algunos estudios dicen que trabajar por tu cuenta lleva a situaciones de estrés ansiedad y soledad, y que gracias a estos espacios han podido sobrevivir a estas épocas de agobios al estar rodeado de profesionales que estén en tu misma situación. Te ayuda a superar tus miedos.

- Networking. Cuando compartes ideas sentimientos anécdotas y sobretodo tiempo con personas que está en la misma situación que tú, la agenda de contactos aumenta de manera de manera muy notable y esto ayuda a tener una amplia red de contactos.

\footnotetext{
${ }^{7} \mathrm{http} / / /$ www.comunidadcoworking.es/que-es-coworking/.
} 
- Huir de las distracciones. En las oficinas de coworking te distraes mucho menos que pudiendo estar en contacto en tu casa donde puedes encontrar millones de distracciones.

- Imagen de profesionalidad. Una gran ventaja de las alas de con working es que existen salas para atender a clientes y proceder a reuniones dentro de ellas. Esto da una imagen de mucha más profesionalidad que si te reúnes en cualquier otro sitio. Ve el ambiente en el que te mueves, y la buena calidad de compañeros que tienes.

- Contagiarse del éxito de otros. Cuando ves que todos los compañeros consiguen sus objetivos, o que tienen grandes y nuevos proyectos, hace que tú quieras satisfacer tanto como ellos. Alegrarse porque por ellos y participar en sus celebraciones crea una alegría interior que hace que tú también quieras conseguirlo.

- Interacción. Puedes encontrar a gente que pueda ayudarte en tus proyectos, o lanzar unos nuevos gracias a la ayuda de esas personas ${ }^{8}$.

- Separar vida profesional y vida privada. Te ayuda a separar la vida profesional con la vida privada, y eso siempre puede conllevar ventajas porque de lo contrario, nunca escaparás de la vida profesional.

Como conclusión de las ventajas que puede ofrecer el coworking, es que se encuentran accesibles frente a la población, siendo estos lugares céntricos; disponen de buena conectividad a internet dentro del recinto, ya que va más enfocada a la conectividad que tienen las personas que forman parte del proyecto; a los integrantes se les facilita asistencia jurídica o financiera, para que realicen sus actividades de emprendimiento y no tengan ningún problema a la hora de lanzar sus ideas; se les ofrecen horarios flexibles, descuentos en estacionamientos, etc.; el coste del alquiler de las oficinas suele ser elevado, pero al realizarlo entre varias personas este coste se ve mermado, por ende, el modelo de negocio se desarrolla y las personas pueden formarse ${ }^{9}$.

\subsection{Inconvenientes del coworking}

Algunas personas señalan ciertos puntos débiles que pueden ir en contra de esta nueva tendencia. Explican que quizás el auge de este nuevo concepto sea más debido a la posibilidad de encontrar locales con un alquiler más flexible y como una solución a la soledad de los trabajadores, más que a aportar valor acorde con la esencia del coworking. Estos espacios no tienen por qué servir a todos los trabajadores si son lugares que resultan ruidosos o divertidos ${ }^{10}$.

\footnotetext{
${ }^{8} \mathrm{https}: / /$ freelandinnovation.com/.

${ }^{9} \mathrm{https}$ ://coworkingspain.es/magazine/noticias/el-coworking-tiene-su-historia

${ }^{10} \mathrm{https}$ ///freelandinnovation.com/que-coworking-elegir/.
} 
En línea con este último riesgo potencial, también han surgido otras empresas que ofrecen justo lo contrario: un lugar donde concentrarse. Puedo resumir los inconvenientes en:

- Competencia. Cuando hay muchas personas trabajando en el mismo sector, la competencia puede llegar a ser un problema. Más aún si estás sentado o trabajando en la misma zona que todos los que se dedican a lo mismo que tú. Además, puede provocar distracciones.

- Distracciones. Hay diferentes tipos de conworking, entre ellos, Hay algunos en los que el silencio no es la mayor virtud. Esto puede provocar que no puedas dar lo máximo de ti mismo a la hora de trabajar en tu espacio de trabajo compartido. No obstante, hay soluciones como ponerse cascos o tapones. Esto puede evitar el trabajo poco productivo.

- Malos compañeros de trabajo. Como en todos lados puedes encontrarte a personas que te impidan trabajar de la manera que tú quieres. Este problema no es muy común, pues la gente que elige trabajar en este tipo de espacios suele dedicarse a la colaboración en equipo...

- Límite de horarios. Si alguna vez andas pillado de tiempo para completar una cosa urgente, puede que el tiempo en el que esté abierto tu espacio de con working sea un impedimento para finalizarlo.

- Envidia. Al igual que el éxito es compartido la envidia también puede serlo. Siempre va a haber gente que esté interrumpiendo tus objetivos. Para esto hay que mejorar la convivencia y hacer un esfuerzo para no llegar a la frustración.

Además, quisiera añadir que hay que seguir una serie de normas, y estaríamos de acuerdo en que no puedes estar como en tu casa en espacios de trabajo comunes. Hay que trabajar la convivencia para no afectar de manera negativa a otros compañeros. Las reuniones no se pueden alargar porque hay que respetar Los tiempos de trabajo de otras personas y al ser un espacio común, habría problemas si esto no se cumpliese. Además, se pierde la privacidad y puede ser incómodo. 


\subsection{Conclusión parcial}

Se resume en el siguiente cuadro:

\begin{tabular}{|l|l|}
\hline VENTAJAS & INCONVENIENTES \\
\hline Crecimiento de círculos sociales & Poca privacidad \\
\hline Ampliación de redes profesionales & Alta rotación de coworkers \\
\hline Incrementos en la productividad & Control y registro de los accesos \\
\hline $\begin{array}{l}\text { Mejoras en aspectos relativos a la } \\
\text { salud derivados y vida personal }\end{array}$ & $\begin{array}{l}\text { Pérdida de capacidad para centrarse en } \\
\text { el propio proyecto }\end{array}$ \\
\hline Reducción del aislamiento & $\begin{array}{l}\text { No ser dueño de tus herramientas de } \\
\text { trabajo }\end{array}$ \\
\hline Disminución del gasto & \\
\hline Cercanía a los centros & \\
\hline Servicios a los que se tiene acceso & \\
\hline
\end{tabular}

\subsection{Generación de conocimientos y referentes}

Todas las relaciones del coworking que se generan se articulan en red y provienen de una comunidad de personas ubicada en un espacio físico concreto que, gracias a las nuevas tecnologías de la información y la comunicación, reciben inputs de otras comunidades, además de que generan output para interactuar con otras ubicadas en diferentes lugares.

Por la práctica del coworking, como se realiza a través de la red, se puede acceder desde cualquier momento y lugar, convirtiéndose así en una fuente del saber que esté al alcance de todos. Los documentos, artículos, estudios, datos, etc., provienen de la misma red, es decir, de internet y por tanto, gracias a la colaboración de usuarios a nivel mundial todos obtienen conocimientos y obtiene documentación de primera mano. Un ejemplo de esto podría ser Wikipedia, la cual es escrita y editada por una extensa red de usuarios a nivel mundial que no tienen una forma de afiliación formal con la publicación.

\subsection{Filosofia del coworking}

El coworking es un saber, que parte de algo anterior a sí mismo, es un saber común formado por una serie de principios que evolucionan con el paso de los años, progresando de una índole individual a una colectiva; esto es, 


\begin{tabular}{|l|l|}
\hline COLECTIVO & INDIVIDUAL \\
\hline Colaboración & Competición \\
\hline Comunidad & Agendas \\
\hline Participación & Observación \\
\hline Actuar & Decir \\
\hline Compañerismo & Formalidad \\
\hline Valentía & Seguridad \\
\hline Aprender & Especialidad \\
\hline Personas & Personalidades \\
\hline Ecosistema & Cadena \\
\hline
\end{tabular}

Como podemos observar, en todos estos elementos predomina el carácter personal que se le atribuye a cada uno de ellos, son únicamente atribuibles a las personas ya sean acciones, sentimientos y gestos. Esta filosofía está inspirada en la cultura participativa y el empoderamiento de las nuevas tecnologías, compartiendo vivencias y experiencias de las que siempre aprende, buscando mejorar y evolucionar. Con la realización del coworking se consigue lograr soluciones de manera inesperada, logrando ideas brillantes.

En este orden de cosas, podemos enunciar los siguientes criterios participativos que reflejan valores corporativos Vs. valores coworking y que podemos resumir así:

Valores corporativos

- Somos tu empleador.

- Vendrás a la oficina.

- Te quedarás en tu cubo.

- Hablar con otros compañeros de trabajo lo distrae de su trabajo.

- Trabajarás en cualquier proyecto que pongamos frente a ti.

- Los pondrás en horas de cara, así que sé que estás trabajando y no te estás tirando.

- Tu trabajo y tu vida fuera del trabajo están separados.

Valores de coworking ${ }^{11}$

- Eres mi cliente.

- Fijaré mis propias horas.

- Trabajaré donde mejor me quede.

- Hablar con otras personas energiza mi trabajo, me ayuda a colaborar y resolver problemas, y es esencial para mi bienestar social.

\footnotetext{
${ }^{11} \mathrm{http}$ :/www.comunidadcoworking.es/community-builder-coworking/
} 
- Trabajaré en proyectos que sean significativos para mí.

- Trabajaré hasta completar el proyecto.

- Mi trabajo y mi vida están entrelazados.

\section{PERSONAS USUARIAS VS. PÚBLICO OBJETIVO}

Debemos realizar una diferenciación entre el público objetivo y usuarios efectivos de los centros coworking. En primer lugar, hablaremos del público objetivo, donde hablamos de los autónomos, los freelances, es decir, quienes teletrabajan. Lo que buscan es compartir para hacer el trabajo más atractivo y lo realicen en comunidad. En segundo lugar, hablamos de usuarios que teletrabajan a distancia para grandes o medianas empresas cuya finalidad es integrarse en espacios coworking para ampliar la red de contactos facilitando el cambio cultural y concordar la vida familiar y laboral.

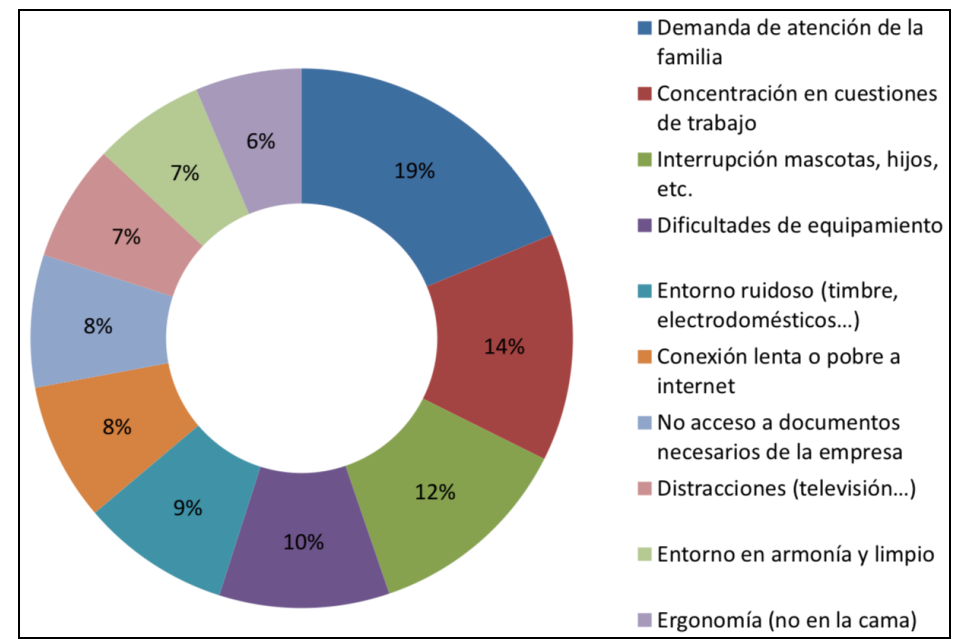

Las personas que desarrollan el teletrabajo lo que buscan con el coworking es compartir ideas y experiencias. Ya que el teletrabajo siempre se ha interpretado como "el trabajo desde casa", es decir, acercar el trabajo a la persona ${ }^{12}$. Pero para que existe el teletrabajo se necesita una conexión telemática entre el equipo y la entidad. El coworking no puede relacionarse con el trabajo que esté vinculado con la mano de obra directa. Los grupos de teletrabajo que podemos encontrar son: trabajador con relación contractual con la empresa; freelance que trabaja por cuenta ajena con terceros, teniendo un carácter autónomo o de autoempleo; combinación entre la relación contractual de la empresa y el trabajo por encargo.

\footnotetext{
${ }^{12} \mathrm{https}: / /$ www.bbva.com/es/que-es-un-hub-de-emprendimiento/
} 
El coworking, por tanto, supone adaptarse a una filosofía y estilo de trabajo concretos, basándose en herramientas y metodologías muy útiles. Es un cambio de mentalidad en cuanto a las actuaciones que se producen en los ámbitos de trabajo. Por tanto, llegamos a la conclusión de que está orientado a personas con estilos de vida diferentes y que lo que buscan es relaciones sociales para compartir su éxito y reconocimiento. Estas personas lo que comparten es la idea de trabajo que tienen, es decir, están interesadas en moverse de manera horizontal pero no vertical. Quieren arremeter diferentes roles y están orientados a entornos y cambios constantes.

Por tanto, lo que busca es fusionar a las personas en diferentes colectivas en un mismo espacio, para obtener una mayor red de nodos que cohesionen entre sí para crear nuevas combinaciones e ideas, para así explorar nuevas oportunidades de negocio y tener la capacidad de reconfigurar las estructuras.

\section{MEJORES ESPACIOS DEL COWORKING EN EL MUNDO}

Se pueden localizar geográficamente con el siguiente criterio:

- Rainmaking Loft, Reino Unido (Londres): emprendedores que utilizan herramientas muy innovadoras para hacer crecer la empresa. Admiten únicamente a personas que están dispuestas a dar todo por su trabajo. El espacio está acondicionado con tumbonas, sofás donde los empleados puedan ver la televisión y descansar, además de futbolines y otros juegos de mesa con los que poder divertirse.

- Sende, Orense (España): es un pequeño pueblo, muy bello, donde puedes realizar sesiones de comunicación, talleres sobre conflicto de refugiados, Porque no es necesario estar en la ciudad rodeado de edificios y atascos, sino que los empleados también necesitan descansar.

- Ziferblat, San Petersburgo (Rusia): es un experimento social donde pagan por el tiempo y no por el espacio. Puedes realizar cualquier actividad, ya sea: leer, tocar el piano, escuchar música, etc.

- Utopic_Us, Madrid (España): ofrece oficinas privadas para que puedas desarrollar todas tus ideas sin que nadie influya en ellas. En este lugar, puedes intercambiar servicios entre diferentes profesionales por las instalaciones que facilita para ello gracias a las escuelas, talleres, galería de arte, gastrobar, etc. 
- Agora Collective, Berlín (Alemania): dispone de un edificio de cinco plantas, donde no solamente trabajas, sino que buscas conectar con otros freelances, emprendedores y artistas de diferentes lugares del mundo. La asociación interpersonal es posible gracias a los diferentes acontecimientos que este espacio de coworking instaura a lo largo de todo el año con películas, musicales, conferencias, prácticas, exposiciones.

- MakersofBarcelona, Barcelona (España): lugar donde te explican a utilizar impresoras 3D y donde poder llevar a cabo tus proyectos, también puedes disfrutar de un café, relajarte y desconectar del portátil.

- GarajeMadrid, Madrid (España): es un espacio que dispone de luz natural, cafetería, futbolín, biblioteca del emprendedor, zona de reuniones informal, chill-out, zonas para los niños. Tiene todo lo necesario para continuar con los proyectos que necesites y nada se te interponga por el camino.

- Coworking in the sun, Islas Canarias (España): lugar donde poder disfrutar de las cálidas y paradisiacas playas de Tenerife, a la vez que trabajas. Este se encuentra dentro de una escuela internacional del marketing a la que acuden estudiantes de toda Europa.

- Bix Spaces, Melbourne (Australia): es un ambiente donde van unidos la profesionalidad y la comunidad y profesionalidad. Dispone de mesas innovadoras y barril gratis de cerveza lo que hace que los autónomos estén disfrutando mientras trabajan.

- Brooklyn Boulders, Massachusetts (Estados Unidos): es uno de los coworking más dinámicos y divertidos. El rocódromo y las máquinas de gimnasia en el lugar de trabajos, como si fuese un patio de colegio para emprendedores con ganas de mantener la forma y divertirse entre reunión y reunión, además de poder disfrutar en sus lugares de trabajo y no solo trabajar. Un rocódromo para realizar escalada puede ser una forma de desconectar del trabajo, asimismo disfrutar con tus compañeros.

Respecto a los resultados vamos a hablar de una revista alemana Deskmag que ha hecho diversos análisis donde ha estudiado el estudio bienal que analiza el sector del coworking a nivel internacional con datos suministrados por los gestores de los espacios y coworkers de todo el mundo. Este fenómeno se ha demostrado que está ayudando a despegar muchos negocios innovadores, y como resultados en las encuestas se ha visto que desde 2013 se ha vuelto a doblar 
en número de espacios donde se emplea la técnica. Además, he podido ver que nuestro país sigue en cabeza de la aplicación de este fenómeno socioeconómico ${ }^{13}$.

Los primeros resultados de Coworking Survey 2015-2016 indican que se ha pasado de 3.400 centros en 80 países a 7.800 en dos años de relativa recuperación económica. En 2011 se contabilizaban sólo 1.130 coworks y en ese momento ya se estaba comentando que era un método esperanzador frente a la crisis que se estaba produciendo principalmente en países como Alemania, Estados Unidos y España, que eran los países de referencia en la implantación y futuro crecimiento de este recurso, y qué a día de hoy lo están clasificando como un nuevo modelo de organización empresarial. España se ha "subido a la ola" de lleno en esta tendencia, la de trabajar en espacios sin paredes, además el portal de referencia de nuestro país sobre este fenómeno ha registrado en 2015 más de 700 coworks y centros de negocios con coworking, cuando las cifras en 2013 eran de 199 espacios. El seguimiento de Alemania es menos exhaustivo que el que se realiza en España.

En los interesantes resultados de la encuesta Global Coworking Survey de Deskmag podemos observar cómo la previsión de aumento de este tipo de espacios es del $22 \%$, que aunque ralentiza suavemente sobre el $30 \%$ anterior, continúa siendo una importante cifra que da idea de la influencia de este tipo de espacios de trabajo dentro de la familia de las diferentes modalidades de Workspaces.

El número de espacios de coworking en todo el mundo ha alcanzado el número de 13.800 puntos, esto confirma que este espacio sigue siendo muy atractivo para muchos profesionales. A parte de estas cifras, se está viendo que los encuestados muestran un interés creciente en que estos espacios sigan creciendo, la cuestión fue realizada en 2016 y se rechazó la idea de si se necesitaban más espacios donde desarrollarlo por un $85 \%$, en cambio en 2017 , este porcentaje ha disminuido bastante hasta un $79 \%$, por lo que vemos que se ha visto un interés mayor por la aplicación de esta herramienta. El coworking es el último gran éxito del mercado laboral, el último taquillazo del sector inmobiliario. La fiebre por los espacios compartidos circunnavega el mundo con obstinación. Es un viaje a través de un cambio de tiempo, tanto para lo bueno como para lo malo, se desvanecen muchas de las ideas que hasta ahora teníamos por concepto de trabajar, el concepto de jornada laboral cambia, las vacaciones. Estos profesionales que la utilizan suelen ser jóvenes, emprendedores y tecnológicos, que buscan espacios que hablen para ellos. Más de 2,3 millones han encontrado el lugar su lugar en el que realizar el coworking. Estos espacios sirven para

\footnotetext{
${ }^{13}$ https:/elpais.com/economia/2018/09/21/actualidad/1537529790_101060.html.
} 
muchas tareas, trabajar, reuniones, presentaciones, comidas, fiestas, etc. Esto cumple el principio de versatilidad que recorre estos nuevos lugares, se mueven dentro de lo industrial y lo cotidiano, por lo que desprenden un sentimiento de comunidad a lo grande.

Pero ¿el coworking es el futuro? ¿O es una moda a corto plazo? La investigadora Gretchen Spreitzer lleva cuatro años estudiando sus efectos. "Creo que esta forma de trabajo será importante para empleados a distancia y freelances porque les da un sentido comunitario del trabajo", explica. Además, sienten que no compiten con nadie. Al contrario, es una herramienta frente a la soledad. Trabajar de forma remota tiene sus ventajas: autonomía, flexibilidad de horarios y control sobre el trabajo. Pero si preguntamos a algunos de estos profesionales se quejaron del aislamiento. En la siguiente imagen podremos ver por zonas geográficas el número de espacios de coworking alrededor del mundo.

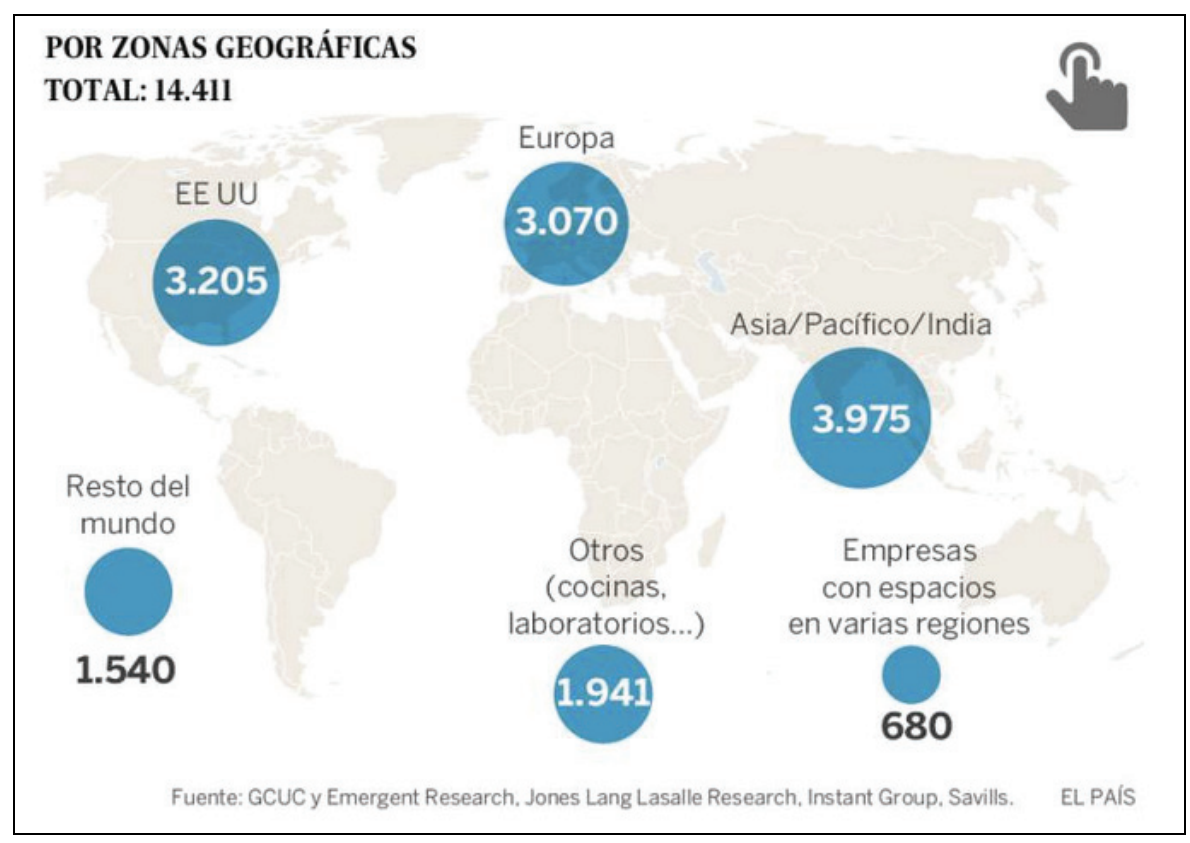

Lo han reconocido como un Imán para crecer, ya que lucha contra las tristezas cotidianas, y para ello están desde las salas comunes hasta los propios inquilinos relatando los proyectos a los compañeros, porque son una comunidad, que hace que crezcan los negocios y se desarrollen nuevas conexiones entre personas de diferentes ámbitos y áreas de conocimiento. 
El coworking quiere cambiar el modelo clásico, y sus números revelan que se ha aumentado desde 2007 hasta el 2018 los espacios de coworking, y que podrá llegar a alcanzar en el 2022 a 5,1 millones de espacios en el mundo. "Esta fórmula solo tiene recorrido en ciudades con mucha oferta de alquiler y en empresas jóvenes que crean empleo y necesitan espacios flexibles a precios razonables", sostiene el emprendedor Rodolfo Carpintier ${ }^{14}$.

Al principio de la crisis de 2008, la precariedad del empleo, las multinacionales despidiendo y los diversos problemas económicos que había en ese periodo complicaba mucho el mundo laboral. La recesión hizo que estos espacios tomarán sentido por el hecho de que era más barato tener a los empleados fuera del despacho, como podrían ser los falsos autónomos que pagan sus propias cotizaciones sociales, por lo que tenía lógica como reducción de costes de estructura de las empresas, y también para el empleo tenía mejor solución que el paro. Por esta razón muchas personas se refugiaron en este estilo de empleo y en fomentar el emprendimiento como alternativa a la inestabilidad que había en ese momento. En principio sí que era una respuesta a la precariedad que se sufría, pro se vio a la larga que gracias a este refugio se crearon salida compartida de protección que daban a la gente un espacio para sacar el máximo de ellos mismos y de su potencial.

Ahora lo que se busca es atraer a las grandes empresas a este movimiento; el coworking corporativo. Según Manuel Zea, fundador de CoworkingSpain.es ${ }^{15}$ : "Ahora mismo es uno de los principales movimientos. En dos sentidos. La llegada de pesos pesados al sector y la atracción hacia estos centros de grandes empresas". Frente a la primera categoría estaría el sector inmobiliario, ya que se abrirán muchos espacios en los mejores lugares de las ciudades, ya que la localización resulta esencial para el negocio.

Por eso vemos que al principio no tuvo muchos resultados porque fue una salida fácil frente a muchas personas que estaban en situaciones complicadas en el sector laboral, pero verdaderamente se ven a largo plazo. Ya que es una evolución del concepto, porque han pasado estos espacios de considerarse informales e ideales para pequeñas empresas y autónomos a espacios con alta calidad con capacidad para dar servicio a empresas con un espíritu innovador. Es una nueva de trabajar y atrae al talento, ya que el modelo de startup es aspiracional. Los jóvenes ya no buscan trabajo en empresas tradicionales que les aseguren una estabilidad, sino que apuestan por empresas modernas y

\footnotetext{
$14 \mathrm{https}: /$ www.inmodiario.com/185/26299/razones-grandes-empresas-estan-apostando-espacioscoworking.html

${ }^{15} \mathrm{https}: / /$ coworkingspain.es/magazine/noticias/el-fenomeno-del-coworking-se-consolida
} 
atractivas que les ofrezcan proyectos apasionantes. Este modelo fomenta la cooperación y la colaboración interna saliéndose de la rutina, ya que comparten con otros profesionales de otros departamentos problemas que pueden ayudarles a afrontar las situaciones de otra manera, y se fomenta la creatividad a diario.

\section{RAZONES PARA TRABAJAR EN UN ESPACIO COWORKING}

Muchas personas tienen miedo a comenzar a trabajar en espacios coworking debido a que no es un concepto de trabajo muy desarrollado aún. Tienen dudas de cuánto van a cobrar, cómo van a encontrar clientes, dónde van a trabajar, etc. Todas estas son las dudas que les surgen, además de que es cambiar completamente el concepto de trabajo que se tenía hasta ahora. Entorno al $80 \%$ de los freelances prefieren trabajar al principio desde sus hogares, pero posteriormente se lanzan a la calle donde poder compartir sus ideas y captar a un mayor número de clientes. Las personas quieren separar su vida profesional de su vida personal, porque en los lugares de trabajo pueden desarrollarse y, además, hablar con otras personas y realizar actividades como tomar un café o jugar al futbolín.

Los espacios coworking se pueden alquilar de forma parcial o completa, por ejemplo, los precios en Madrid están en torno a 25 euros en el caso de alquileres parciales o 120 euros en alquileres completos hasta los 510 en caso de despachos. Pero, antes de pensar en los precios, debe pensar en qué lugar de trabajo quiere estar, es decir, las vistas, la creatividad, los cursos, etc.

El coworking ha supuesto cambios en los espacios de trabajo, debido a que las personas trabajaban de manera autónoma desde sus casas y han pasado a compartir lugares con otros trabajadores, donde pueden intervenir en el desarrollo de conocimientos y opiniones, hablar de sus problemas y de su vida social; además, crear nuevos contactos y lo más importante, sinergias de trabajos que puede llevar a tener diferentes clientes con los que trabajar. La imagen que se les proporciona a los clientes es más profesional, porque al compartir un espacio con otras personas, además de que puedas tener una reunión con ellos en un despacho, una cocina o un salón, donde sea todo acogedor y no sea en tu casa, además de que puedan ver la manera en la que trabajas y los distintos cursos de los que se disponen en el nuevo establecimiento donde te encuentras ${ }^{16}$.

16 https://www.ehu.eus/documents/1393006/6224470/El-coworking.pdf/79395a19-3fd6-4858adc8-795cc8ee355d. 
El coworking obliga a las personas a que se arreglen, marquen unos horarios y permita obtener continuamente nuevos contactos, ya sea con compañeros de trabajo, amigos, clientes, etc. Probablemente cada día pienses que has aprendido algo nuevo debido al intercambio de conocimientos con tus compañeros en el entorno de trabajo donde te mueves.

\section{MARKETING Y EL COWORKING}

Para poder incorporar a futuros coworkers es necesario tener conocimientos de marketing, para ser capaces de realizar técnicas y estrategias de promoción y difusión para poder captar a públicos objetivos de manera creativa e innovadora. A través de la radio, televisión, blogs, diarios, publicidad, jornadas de puertas abiertas, etc. Con el fin de atrapar a futuros coworkers para saber cómo será el lugar donde pasarán tiempo y las facilidades con las que contarán. Podrán contemplar las diferentes salas de las que disponen, además de la ubicación, conexión a internet, los eventos, talleres, etc. Por ello es importante que estas personas les dediquen tiempo para tener esa mentalidad abierta para poder ser capaces de cambiar de espacios, y poder habilitarlos para las necesidades que se tengan en ese momento.

\section{FINANCIACIÓN Y RENTABILIDAD}

Deskmag con la colaboración de otros centros de coworking a nivel mundial, buscaron sintetizar y analizar de manera gráfica todos los centros de coworking y los coworkers que podemos encontrar. Cuando las personas deciden crear espacios de este tipo, la preocupación principal de todos ellos es la obtención de recaudar el capital suficiente para ello. Esto se debe a que el capital es necesario para la fase de planificación y el punto de partida de la idea está en torno a 45.000 euros en Europa y 58.000 dólares en EE. UU.

Los espacios de coworking se financian con capital propio, normalmente suelen estar formados por dos o tres personas con diez miembros o menos. A partir de los cincuenta miembros los gestores suelen ser cuatro aproximadamente. El 12\% de estos centros se conforman como una organización sin ánimo de lucro y un sólo porcentaje marginal está gestionado por entidades gubernamentales. Los ingresos que obtienen están en torno al $60 \%$ del propio puesto de trabajo. Pero también los ingresos provienen del alquiler de los espacios, de los eventos, comidas, etc. Todos estos ingresos significan un porcentaje para la compañía, que, junto con la cartera de usuarios iniciales y la financiación, se convertirán en los puntos clave de la planificación estratégica para el desarrollo y la creación de 
espacios coworking. Por ello, llegamos a la conclusión de que cuanto más grande sea el espacio, mayor rentabilidad se obtendrá del alquiler de las salas de eventos, reuniones, además de los espacios para asentar escritores para coworkers $^{17}$.

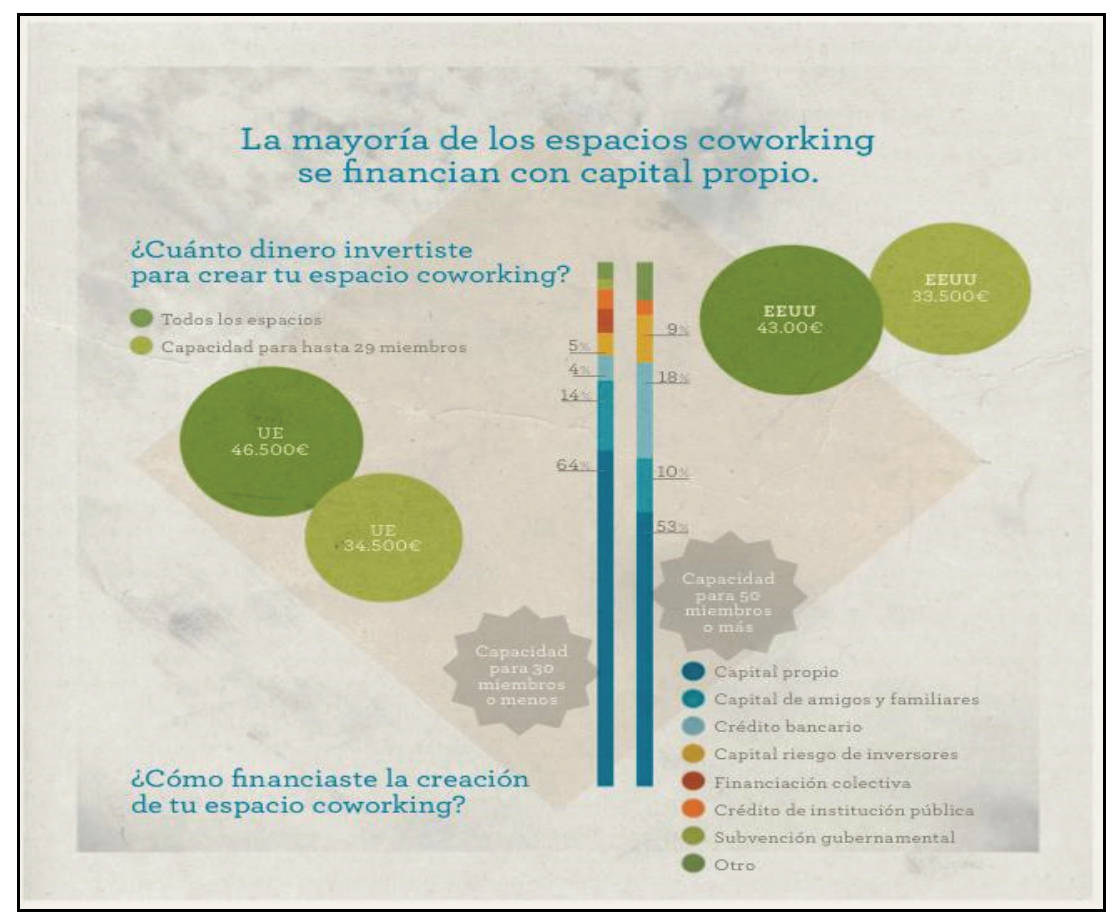

\section{IX.ELCOWORKING EN SUCURSALES BANCARIAS}

El sector financiero se caracteriza por ser muy innovador, suelen tener las últimas tendencias en tecnología. En los últimos años la banca física está pasando a ser banca digital, esto hace que el cliente se haga la gran mayoría de gestiones, pueda visionar sus cuentas, etc. Podemos hablar de las bancas Fintech, estas aprovechan las tecnologías al máximo permitiendo una mejora de los servicios financieros a empresas y particulares. Éstas permiten conocer los clientes, optimizar los servicios de pago y transferencias, el desarrollo de inversiones. Como estamos observando, la banca está evolucionando en todos sus aspectos, tanto en la gestión como en sus oficinas. La innovación está haciendo que las sucursales den otros usos.

17 https://proworkspaces.net/es/deskmag-publica-los-primeros-resultados-la-nueva-encuesta-globalcoworking-2017/. 
Una de las noticias lanzadas por el Banco Santander, anunció que abriría espacios coworking en sus oficinas. Aplicarán técnicas desing thinking para identificar nuevos usos de las oficinas bancarias. Lo que buscan es innovar y cambiar el sector financiero concretamente en Chile, lo que busca es poner todos los servicios que tiene al servicio de los demás. Lo que están aportando es que la zona donde se instaure se construyan redes, se construyan una comunidad, siendo un 50\% banco, $50 \%$ café y co-work. La gente está encontrando no solo venir a tomarse un café, sino que puedan trabajar con otras personas y puedan encontrarse con alguien. En conclusión, lo que se busca es acercar la banca al público en general, además de una atención cercana y profesional generando confianza en todo momento. "La entidad ha iniciado un proceso de transformación en sus oficinas por el que habilitará espacios de 'coworking' para clientes y no clientes de la entidad con el objetivo de reforzar su imagen de marca"18. Además de que, en Madrid en el año 2015, abrió la primera sucursal "Smart Red" en la calle Cea Bermúdez introduciendo canales digitales en los espacios tradicionales. El introducir estos espacios en sus oficinas es una tendencia al alza para la compañía, duplicando casi las proyecciones para 2020 en unos 30.000 a nivel mundial. Lo último instaurado en Madrid ha sido el Work Café, un novedoso espacio al que puede acceder cualquier tipo de público, es decir, clientes como no clientes. La entidad de Santander dice que "agrupa en un solo lugar banco, cowork y cafetería". "Es el reflejo de una nueva sociedad, que busca espacios flexibles y cómodos en los que poder relacionarse e intercambiar ideas y proyectos", apuntan desde Santander, entidad que recientemente remodeló su imagen corporativa para adaptarse a los nuevos tiempos.

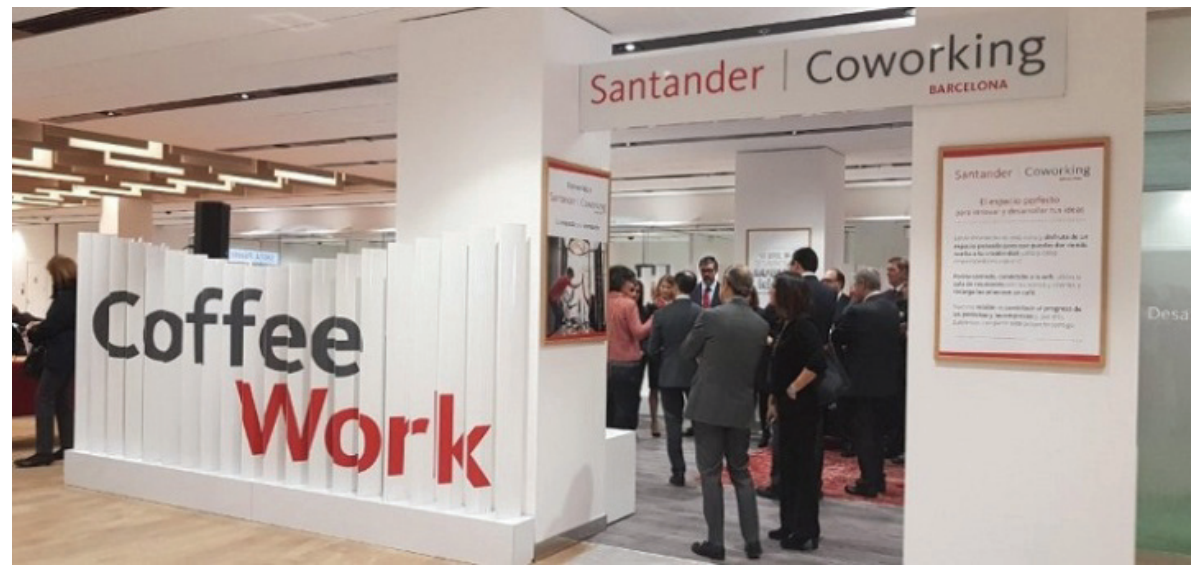

\footnotetext{
${ }^{18} \mathrm{http} / / /$ www.pacoprieto.com/de-sucursal-bancaria-a-espacio-de-coworking.html.
} 
Banco Sabadell, también se ha unido a iniciativas similares con TSB, su filial en Reino Unido, con espacios compartidos donde los trabajadores de servicios centrales desarrollan su actividad. Frente a la catedral de Milán hay un banco llamado Intesa Sanpaolo, este parece antes una pastelería a un banco; en este, puedes sacar tanto dinero como pedir un pastel. La idea gusta a los clientes que tienen el dinero en este banco, además es una buena razón para ir al banco a realizar cualquier gestión, porque dispone de la opción de tomarlo allí o pedirlo para llevar.

Este nuevo tipo de iniciativas también está extendiéndose en otros países. Banco Galicia (Argentina) y otros bancos en Canadá, por ejemplo, cuenta con un banco café disponible también tanto como para clientes como para no clientes y es un espacio donde se combina el servicio de cafetería con la atención de los gestores de banca comercial. Se puede charlar sobre finanzas, wifi gratis, disfrute de asistencia a eventos...etc.

El caso más llamativo que hemos encontrado ha sido el de Repsol, Antonio Pérez Lepe habló de este proyecto que ha introducido a la compañía al mundo del coworking, y que su objetivo es la apertura de la corporación, es crear espacios o entornos de trabajo más creativos y enriquecedores ${ }^{19}$. La iniciativa fue fruto de una campaña que se lanzó orientada a descubrir alguna nueva fórmula de trabajo innovadores, por lo que se centró en la deslocalización del empleado. Y se dieron cuenta que tenía una ventaja clara que era la retención del talento, ya que si un empleado se cambiaba de ciudad no implicaría que tendría que abandonar la empresa. Se hace periódicamente un control de que los empleados acudan a estos espacios de coworking para que puedan nutrirse del ecosistema del emprendedor y trasladar a la compañía nuevos puntos de vista o perspectivas relacionadas con la sociedad corporativa. La técnica que se utiliza es que el empleado seleccionará el espacio coworking y estará rotando durante 6 meses, y tendrá que realizar un feedback, como por ejemplo del tipo de acciones de networking que se lleve a cabo y de las relaciones que surjan a través de las redes. El objetivo es que este sea una modalidad de trabajo en un futuro. Así el cambio de mentalidad está presente en el uso interno de ciertas herramientas orientando a mejorar el intercambio de conocimiento y de la comunicación, que también fomentará la vocación colaborativa y generadora de transversalidad dentro de la corporación.

Otras empresas siguen el mismo modelo como son ATT, Barclays, Orange e incluso Endesa, que recientemente ha comunicado la creación de un espacio de trabajo colaborativo para impulsar su transformación digital y mejorar la

\footnotetext{
${ }^{19}$ https://elpais.com/economia/2018/09/21/actualidad/1537529790_101060.html.
} 
imagen de marca. También General Electric ha planteado diseñar zonas en su nueva sede en Boston accesibles para profesionales y empresas innovadoras externas, con el fin de fomentar la creatividad, el aprendizaje, la colaboración y en definitiva, la innovación.

\section{CONCLUSIÓN}

Coworking es una filosofia de trabajo y de vida que permite a profesionales de diferentes sectores, compartir un mismo espacio de trabajo sin perder su independencia.

Algunas de las palabras que mejor definen estos conceptos son:

- Comunidad: conjunto de personas con vínculos e intereses en común.

- Espacio físico: lugar en el que esas personas desarrollan su actividad y se relacionan.

- Red: relaciones $360^{\circ}$, es decir, todos con todos.

- Interacción social: comunicación entre personas e intercambio de ideas, conocimientos y experiencias.

- TIC's: tecnología de la información y de la comunicación.

- Filosofía: estilo de vida, modo de actuar, ideales a alcanzar, reglas de juego su participación.

- Aprender: entender que de toda experiencia y situación se puede extraer un nuevo conocimiento.

- Feedback: retorno y respuesta tras la escucha activa.

- Unoffice: una oficina, pero no tradicional.

- Profesionales independientes

Finalmente, el coworking puede, y debe, entenderse como un producto o servicio formado por una parte tangible y otra intangible que, en lugar de ser un producto o servicio producido de forma ajena para quienes gestionan, se produce por cuenta propia y donde el gestor decide de qué manera vendérselo; es como si los coworker estuviesen, en parte, pagando por el resultado y las creaciones que son fruto de su propia fuerza de trabajo; están pagando por la compra de parte de su trabajo diario y por formar parte de un producto final ${ }^{20}$.

Lleva a pensar que las personas usuarias de estos centros son la parte clave del producto final, el verdadero valor añadido. La suma del espacio físico y las personas que lo integran, son en sí el producto que quienes los gestionan quieren

\footnotetext{
${ }^{20} \mathrm{https}$ //coworkingspain.es/magazine/noticias/el-coworking-tiene-su-historia.
} 
obtener; es decir el coworking como mercancía. Al aparecer nuevas fórmulas de organización del trabajo y reunión de personas para aprovechar lo mejor de cada parte supone un cambio positivo, siendo punto de partida hacia la investigación y el análisis que puede facilitar la mejora de la vida de las personas y su forma de concebirla. Unos lugares de reunión que se materializan por ejemplo en centros coworking y que son capaces de reunir de forma física a personas de diversas disciplinas creando un nuevo estilo de vida y trabajo, donde se trata de dar un salto y sobrepasar la conciliación para entrelazar ambos ámbitos para que, aun siendo parcelas diferentes de la vida de una persona, puedan convivir juntas sin detrimento la una de la otra. Unos centros que representan nuevas formas de organización poniendo en práctica novedosas reglas de juego que posiblemente extiendan su influencia a otros ámbitos de la sociedad general. "Dentro de 20 años utilizaremos un modelo de trabajo totalmente flexible, y dejaremos de tener un puesto fijo para trabajar en el entorno más adecuado a cada momento y con unos horarios adaptados a las necesidades individuales", vaticina Philippe Jiménez, responsable en España de la firma de coworking $\mathrm{IWG}^{21}$.

"Ver a gente trabajando duro motiva a hacer lo mismo, pero además nunca nos interrumpen, porque nuestro trabajo es independiente, no interdependiente", apunta Ethan Bernstein, profesor de liderazgo de Harvard Business School ${ }^{22}$.

\section{WEBGRAFÍA}

- AIZEGA ZUBILlAGA, J., M., La utilización extrafiscal de los tributos y los principios de justicia tributaria, Bilbao 2001.

- https://www.muypymes.com/2016/02/11/utopic_us

- https://www.utopicus.es

- https://hipertextual.com/2011/05/hackerspaces-refugios-de-creatividad-yconocimiento

- https://definicion.de/freelance/

- http://www.comunidadcoworking.es/que-es-coworking/

- https://www.skyscanner.es/noticias/inspiracion/los-15-espacios-decoworking-mas-cool-del-mundo

- https://riull.ull.es/xmlui/bitstream/handle/915/2851/Espacios\%20Coworki ng.pdf? sequence $=1 \&$ isAllowed $=y$

\footnotetext{
${ }^{21} \mathrm{http} / / /$ www.expansion.com/empresas/banca/2018/04/15/5ad32161e2704e4c298b458c.html.
}

${ }^{22} \mathrm{https}: / /$ cincodias.elpais.com/cincodias/2018/01/04/companias/1515092822_942595.html. 
- https://www.ehu.eus/documents/1393006/6224470/Elcoworking.pdf/79395a19-3fd6-4858-adc8-795cc8ee355d

- http://www.pacoprieto.com/de-sucursal-bancaria-a-espacio-de-coworking.html

- http://www.expansion.com/empresas/banca/2018/04/15/5ad32161e2704e4 c298b458c.html

- https://www.youtube.com/watch? $\mathrm{v}=\mathrm{xrmLPrYKqEU}$

- https://cincodias.elpais.com/cincodias/2018/01/04/companias/1515092822 942595.html

- $\overline{\mathrm{h} t t p}: / /$ players.brightcove.net/55814260001/SyGj52bf_default/index.html?v ideoId $=5751245526001 \#$

- https://www.capital.es/2019/02/26/sofas-cafes-y-salas-de-coworking-paraque-los-millennials-vayan-al-banco/

- http://www.comunidadcoworking.es/que-es-coworking/

- https://freelandinnovation.com/

- https://coworkingspain.es/magazine/noticias/el-coworking-tiene-su-historia

- https://freelandinnovation.com/que-coworking-elegir/

- http://www.comunidadcoworking.es/community-builder-coworking/

- https://www.bbva.com/es/que-es-un-hub-de-emprendimiento/

- https://www.holded.com/es/blog/coworking-ventajas-inconvenientesemprendedores/

- https://www.eleconomista.es/emprendedores-pymes/noticias/5840950/06/14/ Que-es-el-coworking-Ventajas-e-inconvenientes-de-compartir-oficina.html

- https://diarioformacion.wordpress.com/2017/03/23/ventajas-e-inconvenientes -del-coworking/

- https://www.europapress.es/economia/finanzas-00340/noticia-santanderimpulsa-espacios-coworking-oficinas-reforzar-imagen-marca-20180415 114434.html

- https://coworkingspain.es/magazine/noticias/el-coworking-tiene-su-historia

- https://www.ehu.eus/documents/1393006/6224470/Elcoworking.pdf/79395a19-3fd6-4858-adc8-795cc8ee355d

- https://proworkspaces.net/es/deskmag-publica-los-primeros-resultados-lanueva-encuesta-global-coworking-2017/

- https://coworkingspain.es/magazine/noticias/el-fenomeno-del-coworkingse-consolida

- https://elpais.com/economia/2018/09/21/actualidad/1537529790_101060.html

- https://www.inmodiario.com/185/26299/razones-grandes-empresas-estanapostando-espacios-coworking.html 
